\title{
Fibrose cística em um centro de referência no Brasil: características clínicas e laboratoriais de 104 pacientes e sua associação com o genótipo e a gravidade da doença
}

\author{
Cystic fibrosis at a Brazilian center of excellence: clinical and laboratory characteristics \\ of 104 patients and their association with genotype and disease severity
}

\author{
Alfonso E. Alvarez ${ }^{1}$, Antônio F. Ribeiro², Gabriel Hessel ${ }^{3}$, Carmen S. Bertuzzo ${ }^{4}$, José D. Ribeiro ${ }^{5}$
}

\section{Resumo}

Objetivo: Estudar as características clínicas, laboratoriais e radiográficas de pacientes fibrocísticos acompanhados na última década do século 20 na UNICAMP e verificar se existe associação com o genótipo e a gravidade da doença medida pelo escore de Shwachman.

Métodos: Estudo descritivo, retrospectivo e de corte transversal dos pacientes fibrocísticos acompanhados na UNICAMP, que tiveram atendimento entre julho de 1990 e julho de 2000.

Resultados: Foram estudados 104 pacientes: sexo masculino $53,8 \%$; raça caucasóide - $93,3 \%$; comprometimento pulmonar $89,4 \%$, comprometimento digestivo - 59,6\%; íleo meconial - 5,8\%; diabetes melito - 4,8\%; mediana da idade de início dos sintomas 3 meses; mediana da idade no diagnóstico - 2 anos e 4 meses; 69,9 e $56,6 \%$ apresentavam peso e estatura abaixo do percentil 10 , respectivamente, na época do diagnóstico; dosagem de cloro no suor $<60 \mathrm{mEq} / \mathrm{l}-10,6 \%$; colonização: S. aureus - 80,2\%, P. aeruginosa - 76,0\%, B. cepacia - 5,2\%; $\Delta \mathrm{F} 508$ homozigoto - 18,75\%, $\Delta \mathrm{F} 508$ heterozigoto - $62,5 \%$; escore de Shwachman moderado/grave $15,7 \%$. Foram a óbito 18 pacientes $(17,3 \%)$; mediana de idade do óbito de 7 anos e 8 meses; sobrevida mediana após o diagnóstico no término do estudo de 18 anos e 4 meses. Os pacientes com a mutação $\Delta \mathrm{F} 508$ apresentaram balanço de gordura nas fezes alterado com maior freqüência que os pacientes sem essa mutação $(p<0,05)$. Quando comparados os pacientes que apresentavam uma ou duas mutações $\Delta \mathrm{F} 508$, nenhum parâmetro apresentou diferença estatisticamente significativa.

Conclusões: As características clínicas e laboratoriais dos pacientes estudados foram semelhantes às descritas na população fibrocística de outros países, com algumas exceções, dentre as quais destacamos maior idade no diagnóstico e menor sobrevida. Desta forma, nossos dados permitem inferir que esforços para um diagnóstico precoce e maior oportunidade de tratamento necessitam ser dirigidos aos pacientes fibrocísticos.

J Pediatr (Rio J). 2004;80(5):371-9: Fibrose cística, $\Delta$ F508, relação genótipo-fenótipo, Pseudomonas aeruginosa, Burkholderia cepacia, escore de Shwachman, doenças pulmonares pediátricas.

\begin{abstract}
Objective: To identify the clinical, laboratory and radiographic characteristics of the cystic fibrosis patients under care at Universidade Estadual de Campinas (UNICAMP) in the last decade of the twentieth century, and to investigate the association of these characteristics with genotype and severity of the disease as measured by the Shwachman score.
\end{abstract}

Methods: Descriptive, retrospective and cross-sectional study of the patients assisted at UNICAMP hospital's Cystic Fibrosis Clinic from July 1990 to July 2000.

Results: One hundred and four patients were studied; $53.8 \%$ male; $93.3 \%$ Caucasian; $89.4 \%$ presented with respiratory symptoms; $59.6 \%$ presented with digestive symptoms; $5.8 \%$ had meconium ileus; $4.8 \%$ had diabetes. The mean age at onset of symptoms was 3 months, and the mean age at diagnosis was 2 years and 4 months. At diagnosis, 69.9 and $56.6 \%$ of the patients had weight and height below $10^{\text {th }}$ percentile, respectively; in $10.6 \%$, sweat chloride was $<60 \mathrm{mEq} / \mathrm{l}$. Staphylococcus aureus was found in $80.2 \%$, Pseudomonas aeruginosa in $76.0 \%$, and Burkholderia cepacia in $5.2 \%$. $\Delta \mathrm{F} 508$ homozygosis was observed in $18.75 \%$, whereas $62.50 \%$ of the patients were $\Delta$ F508 heterozygous. A moderate/severe Shwachman score was found in $15.7 \%$. Eighteen patients died in that period $(17.3 \%)$. The mean age at death was 7 years and 8 months; median survival after diagnosis was 18 years and 4 months. Patients who have at least one $\Delta \mathrm{F} 508$ mutation have more frequent alterations in fecal fat levels when compared to patients who do not have this mutation $(p<0.05)$. There were no differences in any parameter between $\Delta \mathrm{F} 508$ homozygous and heterozygous patients.

Conclusions: The clinical and laboratory characteristics of the 104 patients studied were similar to the characteristics described for patients in other countries. Exceptions are the higher age at diagnosis and lower survival. Our results support the recommendation for early diagnosis and the need for more treatment opportunities in the population of cystic fibrosis patients.

J Pediatr (Rio J). 2004;80(5):371-9: Cystic fibrosis, $\Delta \mathrm{F508,}$ genotype/phenotype relation, Pseudomonas aeruginosa, Burkholderia cepacia, Shwachman score, pediatric lung disease.

\footnotetext{
1. Mestre; Pneumologista pediátrico; Doutorando em Pediatria, Setor de Pneumologia Pediátrica, Faculdade de Ciências Médicas, Universidade Estadual de Campinas (UNICAMP), Campinas, SP.

2. Doutor; Gastroenterologista pediátrico; Chefe do Dep. de Pediatria; Docente, Setor de Gastroenterologia Pediátrica, FCM, UNICAMP, Campinas, SP.

3. Doutor; Gastroenterologista pediátrico; Docente, Dep. de Pediatria, Setor de Gastroenterologia Pediátrica, FCM, UNICAMP, Campinas, SP.

4. Doutora; Médica geneticista; Docente, Departamento de Genética, Faculdade de Ciências Médicas, UNICAMP, Campinas, SP.

5. Doutor; Pneumologista pediátrico; Docente, Dep. de Pediatria; Responsável pelo Setor de Pneumologia Pediátrica, FCM, UNICAMP, Campinas, SP.
}

Artigo submetido em 22.01.04, aceito em 07.07.04.

Como citar este artigo: Alvarez AE, Ribeiro AF, Hessel G, Bertuzzo CS, Ribeiro JD. Fibrose cística em um centro de referência no Brasil: características clínicas e laboratoriais de 104 pacientes e sua associação com o genótipo e a gravidade da doença. J Pediatr (Rio J). 2004;80:371-9. 


\section{Introdução}

A fibrose cística (FC) tem sido amplamente estudada por autores de diversos países, possibilitando maior entendimento em relação à sua fisiopatologia e o advento de novas modalidades terapêuticas, o que se reflete de maneira significativa na diminuição da morbidade e no aumento da sobrevida dos pacientes.

Existem, porém, poucos trabalhos analisando a população de fibrocísticos dos países em desenvolvimento. Desta forma, o tratamento e as medidas de saúde pública oferecidos aos fibrocísticos de tais países são baseados em dados internacionais, sem se levar em conta suas peculiaridades. Além de tratar tais pacientes sem o conhecimento real de suas características, esta realidade tem outras implicações, pois, no momento em que é necessário escolher as medicações que o Estado fornecerá (na impossibilidade de oferecer todas as necessárias) seria fundamental basear a escolha nas características da população local.

A Organização Mundial de Saúde chamou a atenção para este fato em $1997^{1}$, recomendando as seguintes ações nos serviços de atendimento aos fibrocísticos dos países em desenvolvimento: triagem neonatal para determinar a incidência e identificar os recém-nascidos afetados; implementação dos laboratórios para identificar as mutações da FC; desenvolvimento de centros de diagnóstico e tratamento com equipe multidisciplinar; estabelecimento de uma organização nacional envolvendo familiares, amigos dos fibrocísticos e outros voluntários; estabelecimento de um registro nacional; divulgação sobre esta condição para profissionais de saúde, autoridades públicas e população geral; e aumento da colaboração entre os grupos e outras organizações (incluindo a indústria farmacêutica).

No Brasil, a falta de informações é evidente, existindo poucos trabalhos voltados para a descrição e análise das características dos fibrocísticos.

O objetivo deste estudo foi descrever as características clínicas e laboratoriais de pacientes fibrocísticos acompanhados no Ambulatório de Fibrose Cística do Hospital da Universidade Estadual de Campinas (UNICAMP) na última década do século 20 e verificar sua associação com a presença da mutação $\Delta \mathrm{F} 508$ e a gravidade da doença medida pelo escore de Shwachman (ES).

\section{Casuística e métodos}

Realizou-se um estudo descritivo, retrospectivo e de corte transversal dos pacientes acompanhados no Ambulatório de Fibrose Cística da UNICAMP, atendidos entre julho de 1990 e julho de 2000.

Foram incluídos todos os pacientes que realizaram pelo menos uma consulta no ambulatório e que tiveram o diagnóstico confirmado por história clínica e pelo menos dois testes de suor com valores de cloro igual ou maior que $60 \mathrm{mEq} / \mathrm{l}$, ou pela identificação de duas mutações.

A dosagem de eletrólitos no suor foi realizada através do estímulo do suor pela iontoforese com pilocarpina².

As culturas de escarro foram obtidas para identificação de Staphylococcus aureus, Pseudomonas aeruginosa, Pseu- domonas aeruginosa mucosa e Burkholderia cepacia. As amostras foram semeadas em ágar chocolate, ágar sangue, ágar suplementado, meio de cultura MacConkey (específico para Pseudomonas) e tioglicolato. As cepas mucosas de Pseudomonas aeruginosa foram identificadas visualmente pela morfologia característica (presença de muco líquido). Não foram utilizados meios seletivos para Burkholderia cepacia.

Foram pesquisadas as seguintes mutações: $\Delta \mathrm{F} 508$, G542X, N1303K, G551D, R553X e W1282X. A deteç̧ão da mutação $\Delta \mathrm{F} 508$ foi realizada através de reação em cadeia da polimerase (PCR) e análise em gel de poliacrilamida $8 \%$, com a modificação descrita por Rommens et al. ${ }^{3}$. As outras mutações foram analisadas pela técnica de PCR associada à digestão enzimático-específica.

Para a análise descritiva, utilizaram-se os valores de média, mediana e desvio padrão para as variáveis contínuas e freqüência absoluta para as variáveis discretas. Para a comparação de variáveis categóricas, foi utilizado o teste de qui-quadrado. O teste exato de Fisher foi aplicado nos casos em que uma das células $2 \times 2$ era menor ou igual a 5. Para comparar medidas contínuas ou ordenáveis entre dois grupos, utilizou-se o teste de MannWhitney. A análise do tempo de sobrevida foi realizada pelo método de Kaplan-Meier. Para a comparação das curvas, foi aplicado o teste de Wilcoxon (Breslow). O nível de significância adotado foi de $5 \%$.

A pesquisa foi aprovada pelo Comitê de Ética em Pesquisa da UNICAMP.

\section{Resultados}

Foram estudados 104 pacientes, sendo $53,8 \%$ masculinos, 93,3\% caucasóides e 6,7\% negróides. Dezoito pacientes foram a óbito no período compreendido pelo estudo. Consangüinidade entre os pais ocorreu em $6,2 \%$ da população estudada.

Manifestações respiratórias e digestivas ocorreram em 89,4 e $59,6 \%$ dos pacientes, respectivamente. Seis apresentaram antecedente de íleo meconial e cinco evoluíram com diabetes melito.

A idade referida de início dos sintomas variou desde o nascimento até 20 anos, com média de 16 meses e mediana de 3 meses (Figura 1).

A idade no diagnóstico variou desde o período neonatal até 29 anos e 11 meses, com média de 4 anos e 2 meses e mediana de 2 anos e 4 meses (Figura 2).

Em relação ao estado nutricional, 69,9 e 56,6\% dos pacientes apresentavam peso e estatura abaixo do percentil 10, respectivamente, por ocasião da primeira consulta.

A saturação transcutânea da hemoglobina pelo oxigênio em ar ambiente foi maior que $95 \%$ em 59,5\%, entre 91 e $95 \%$ em $32,9 \%$, e menor que $91 \%$ em $7,6 \%$ dos pacientes.

A dosagem de cloro no suor foi menor que $60 \mathrm{mEq} / \mathrm{l} \mathrm{em}$ $10,6 \%$ (11) dos pacientes, sendo que 1,9\% (2) apresentaram valores abaixo de $40 \mathrm{mEq} / \mathrm{l} ; 28,8 \%$ (30) dos pacientes apresentaram valores entre 60 e $100 \mathrm{mEq} / \mathrm{l}$, e 60\% (63) apresentaram valores acima de $100 \mathrm{mEq} / \mathrm{l}$. 


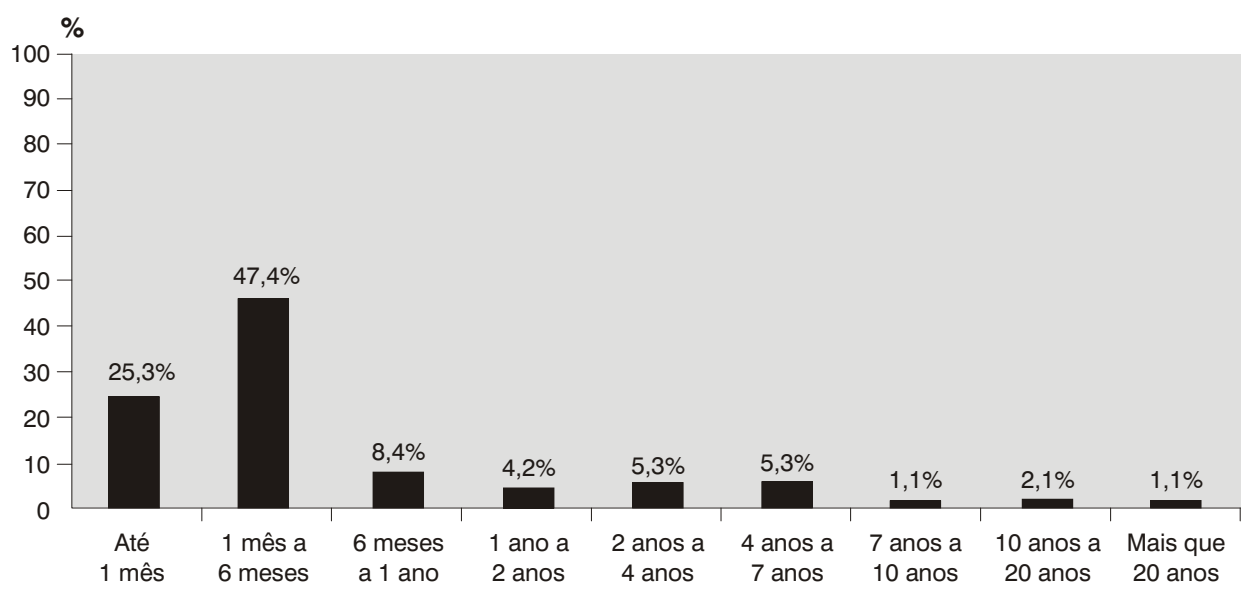

Figura 1 - Distribuição dos pacientes segundo a idade de início dos sintomas ( $n=95)$

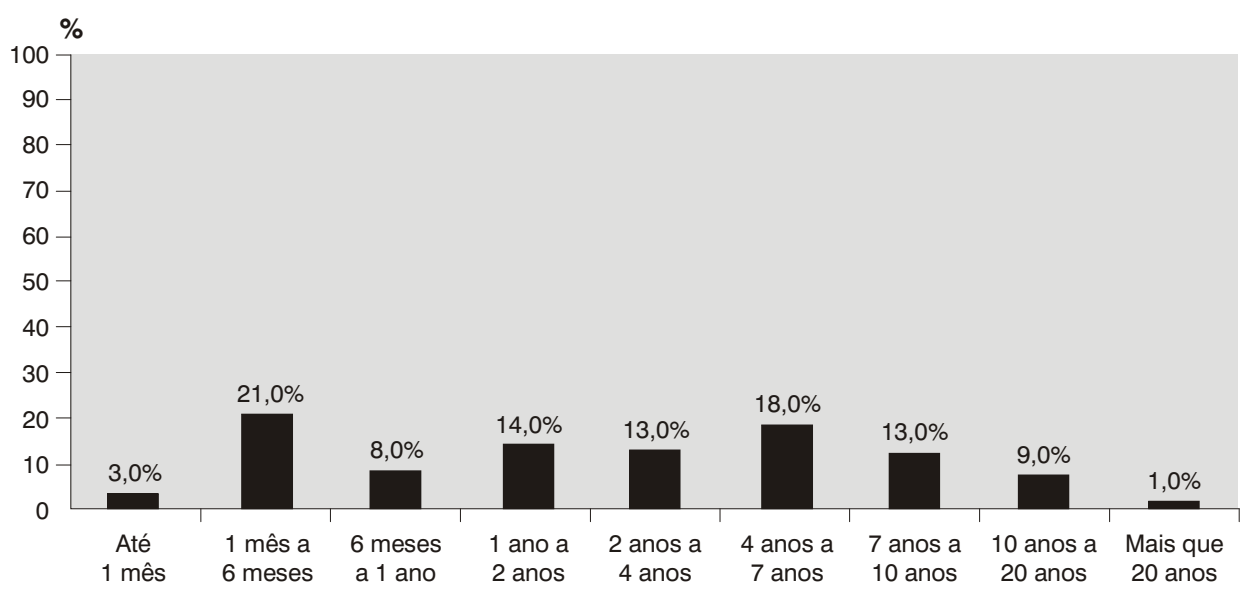

Figura 2 - Distribuição dos pacientes segundo a idade no diagnóstico $(n=100)$

Mais de $80 \%$ estavam colonizados por Staphylococcus aureus, $76 \%$ por Pseudomonas aeruginosa, mais da metade por Pseudomonas aeruginosa mucosa, e 5,2\% por Burkholderia cepacia (Tabela 1).

Tabela 1 - Distribuição dos pacientes segundo colonização pelas diferentes bactérias $(n=96)$

\begin{tabular}{lcc}
\hline Colonização & \% & $\mathbf{n}$ \\
\hline Staphylococcus aureus & 80,2 & 77 \\
Pseudomonas aeruginosa & 76,0 & 73 \\
Pseudomonas aeruginosa mucosa & 53,1 & 51 \\
Burkholderia cepacia & 5,2 & 5 \\
P. aeruginosa e P. aeruginosa mucosa & 51,0 & 49 \\
\hline
\end{tabular}

Espirometria foi realizada em 55 pacientes e apresentava-se normal em $27,3 \%$ (15), com distúrbio ventilatório restritivo em $18,2 \%(10)$, distúrbio ventilatório obstrutivo em $25,4 \%$ (14) e distúrbio ventilatório misto em $29,1 \%$ (16).

Em relação à genética, foram avaliados 96 pacientes, sendo que $18,75 \%$ (18) eram $\Delta \mathrm{F} 508$ homozigotos e $62,5 \%(60)$ eram $\Delta F 508$ heterozigotos. Desta forma, $50 \%$ dos cromossomos estudados apresentavam a mutação $\Delta \mathrm{F} 508$.

Nos 192 cromossomos estudados, encontraram-se seguintes porcentagens de outras mutações: G542X $(4,17 \%), N 1303 \mathrm{~K}(2,08 \%)$, G551D $(1,04 \%)$, R553X $(0,52 \%), W 1282 \times(0,52 \%)$.

O ES foi realizado em 83 pacientes e evidenciou $57,8 \%$ (48) com escore excelente ou bom, 26,5\% (22) com escore médio e $15,7 \%$ (22) com escore moderado ou grave. 
Foram a óbito neste período 18 pacientes (17,3\%), 10 do sexo masculino e oito do feminino, 17 caucasóides e um negróide, todos por insuficiência respiratória.

A idade no óbito variou de 6 meses até 23 anos e 1 mês. A média de idade no óbito foi de 9 anos e 5 meses, e a mediana foi 7 anos e 4 meses.

$\mathrm{Na}$ época do encerramento do estudo, a sobrevida mediana após o diagnóstico era de 18 anos e 4 meses, e $81,39 \%$ dos pacientes sobreviviam 10 anos após o diagnóstico. A curva de sobrevida após o diagnóstico dos pacientes pode ser vista na Figura 3.

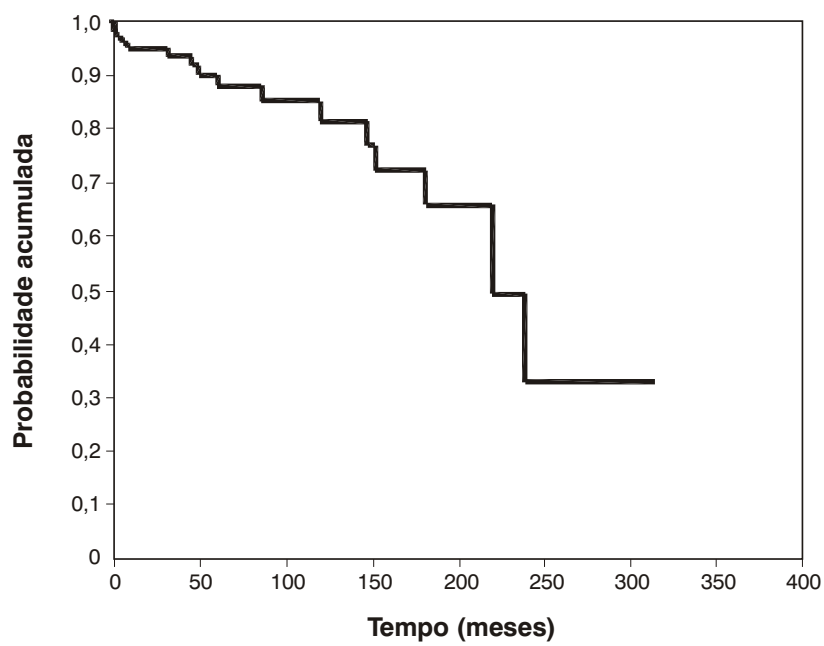

Figura 3 - Curva de sobrevida após o diagnóstico dos pacientes, estimada pelo método de Kaplan-Meier
Procurou-se verificar a correlação do ES com as características clínicas e laboratoriais dos pacientes. Para esta análise, foram incluídos 83 sujeitos, sendo 54,2\% (45) do sexo masculino; $57,8 \%$ (48) apresentavam ES excelente ou bom, 26,5\% (22) médio e 15,7\% (13) moderado ou grave. A Tabela 2 apresenta a correlação estatística dos parâmetros clínicos e laboratoriais com o ES.

Avaliaram-se as diferenças clínicas e laboratoriais entre pacientes fibrocísticos com e sem a presença da mutação $\Delta \mathrm{F} 508$. Foram estudados 96 pacientes, sendo que $81 \%$ (78) apresentavam a mutação $\Delta \mathrm{F} 508$ e $19 \%$ (18) não a apresentavam; 52\% (50) eram do sexo masculino. A Tabela 3 apresenta a correlação estatística dos parâmetros clínicos e laboratoriais com a presença ou não da mutação $\Delta \mathrm{F} 508$.

Para a avaliação de diferenças clínicas e laboratoriais entre pacientes fibrocísticos com a presença da mutação $\Delta \mathrm{F} 508$ na forma homozigota e heterozigota, foram incluídos 78 pacientes, sendo $23 \%$ (18) homozigotos e $77 \%$ (60) heterozigotos para a mutação $\Delta \mathrm{F} 508 ; 47 \%$ (37) do sexo masculino. A presença de homozigose ou heterozigose para a mutação $\Delta \mathrm{F} 508$ não apresentou correlação estatisticamente significativa com nenhum dos parâmetros estudados, conforme apresentado na Tabela 4.

\section{Discussão}

As características clínicas e laboratoriais dos 104 pacientes estudados estão próximas dos valores encontrados em outros trabalhos nacionais e internacionais.

A distribuição em relação a sexo, raça ${ }^{4-6}$ e incidência de manifestações pulmonares $4,7,8$ foi semelhante aos dados encontrados na literatura.

Tabela 2 - Correlação estatística dos parâmetros clínicos e laboratoriais dos pacientes com o escore de Shwachman $(n=83)$

\begin{tabular}{|c|c|c|}
\hline Variáveis & $\mathbf{p}$ & Teste estatístico utilizado \\
\hline Colonização por Pseudomonas aeruginosa & $<0,001$ & Teste exato de Fisher \\
\hline Colonização por $P$. aeruginosa mucosa & $<0,001$ & Qui-quadrado \\
\hline Capacidade vital forçada & $<0,001$ & Teste exato de Fisher \\
\hline Volume expiratório forçado no primeiro segundo & $<0,001$ & Teste exato de Fisher \\
\hline Saturação de oxigênio & $<0,001$ & Teste exato de Fisher \\
\hline Exacerbações infecciosas no último ano & 0,045 & Qui-quadrado \\
\hline Indicação de utilização de Dornase Alfa & 0,019 & Qui-quadrado \\
\hline Indicação de fisioterapia respiratória & 0,017 & Qui-quadrado \\
\hline Indicação de oxigenoterapia domiciliar & $<0,001$ & Qui-quadrado \\
\hline Sexo & 0,810 & Qui-quadrado \\
\hline Dosagem de cloro no suor & 0,656 & Qui-quadrado \\
\hline Colonização por Staphylococcus aureus & 0,058 & Teste exato de Fisher \\
\hline Colonização por Burkholderia cepacia & 0,431 & Qui-quadrado \\
\hline Presença ou não da mutação $\Delta$ F508 & 0,230 & Teste exato de Fisher \\
\hline Presença de uma ou duas mutações $\Delta \mathrm{F} 508$ & 0,669 & Teste exato de Fisher \\
\hline Idade atual & 0,487 & Qui-quadrado \\
\hline
\end{tabular}


Tabela 3 - Correlação estatística dos parâmetros clínicos e laboratoriais dos pacientes com a presença ou não da mutação $\Delta \mathrm{F} 508(\mathrm{n}=96)$

\begin{tabular}{lcc}
\hline Variáveis & p & Teste estatístico utilizado \\
\hline Balanço de gordura nas fezes & 0,005 & Qui-quadrado \\
Sexo & 0,058 & Qui-quadrado \\
Raça & 1,0 & Teste exato de Fisher \\
Presença de manifestações respiratórias & 0,681 & Teste exato de Fisher \\
Presença de manifestações digestivas & 0,079 & Qui-quadrado \\
Antecedente de íleo meconial & 0,579 & Teste exato de Fisher \\
Idade de início dos sintomas & 0,673 & Mann-Whitney \\
Peso atual & 0,368 & Teste exato de Fisher \\
Presença de diabetes melito & 0,570 & Teste exato de Fisher \\
Colonização por Staphylococcus aureus & 0,751 & Teste exato de Fisher \\
Colonização por Pseudomonas aeruginosa & 0,067 & Teste exato de Fisher \\
Colonização por P. aeruginosa mucosa & 0,525 & Qui-quadrado \\
Colonização por Burkholderia cepacia & 1,0 & Teste exato de Fisher \\
Capacidade vital forçada & 0,089 & Teste exato de Fisher \\
Volume expiratório forçado no primeiro segundo & 0,723 & Teste exato de Fisher \\
Presença de bronquiectasias & 1,0 & Teste exato de Fisher \\
Presença de hepatomegalia & 0,327 & Teste exato de Fisher \\
Escore de Shwachman & 0,230 & Teste exato de Fisher \\
Exacerbações infecciosas no último ano & 0,298 & Teste exato de Fisher \\
Indicação de enzimas pancreáticas & 0,265 & Qui-quadrado \\
Indicação de fisioterapia respiratória & 0,351 & Teste exato de Fisher \\
Indicação de Dornase Alfa & 0,540 & Qui-quadrado \\
Indicação de oxigenoterapia domiciliar & 0,255 & Teste exato de Fisher \\
Evolução para óbito & 1,0 & Teste exato de Fisher \\
Idade do óbito & 0,606 & Mann-Whitney \\
Curva de sobrevida & 0,595 & Teste de Wilcoxon \\
\hline
\end{tabular}

Tabela 4 - Correlação estatística dos parâmetros clínicos e laboratoriais dos pacientes com a presença da mutação $\Delta$ F508 na forma homozigota ou heterozigota $(n=78)$

\begin{tabular}{lcc}
\hline Variáveis & $\mathbf{p}$ & Teste estatístico utilizado \\
\hline Sexo & 0,803 & Qui-quadrado \\
Raça & 0,617 & Teste exato de Fisher \\
Presença de manifestações respiratórias & 0,423 & Teste exato de Fisher \\
Presença de manifestações digestivas & 0,966 & Qui-quadrado \\
Antecedente de íleo meconial & 0,325 & Teste exato de Fisher \\
Idade de início dos sintomas & 0,284 & Mann-Whitney \\
Peso atual & 0,570 & Teste exato de Fisher \\
Presença de diabetes melito & 1,0 & Teste exato de Fisher \\
Colonização por Staphylococcus aureus & 0,493 & Teste exato de Fisher \\
Colonização por Pseudomonas aeruginosa & 0,746 & Teste exato de Fisher \\
Colonização por P. aeruginosa mucosa & 0,413 & Qui-quadrado \\
Colonização por Burkholderia cepacia & 0,258 & Teste exato de Fisher \\
Balanço de gordura nas fezes & 1,0 & Teste exato de Fisher \\
Capacidade vital forçada & 0,145 & Teste exato de Fisher \\
Volume expiratório forçado no primeiro segundo & 0,161 & Teste exato de Fisher \\
Presença de bronquiectasias & 0,646 & Teste exato de Fisher \\
Presença de hepatomegalia & 0,734 & Teste exato de Fisher \\
Escore de Shwachman & 0,669 & Teste exato de Fisher \\
Exacerbações infecciosas no último ano & 0,835 & Teste exato de Fisher \\
Indicação de enzimas pancreáticas & 0,529 & Teste exato de Fisher \\
Indicação de fisioterapia respiratória & 1,0 & Teste exato de Fisher \\
Indicação de Dornase Alfa & 0,051 & Qui-quadrado \\
Indicação de oxigenoterapia domiciliar & 1,0 & Teste exato de Fisher \\
Evolução para óbito & 0,227 & Teste exato de Fisher \\
Idade do óbito & 0,352 & Mann-Whitney \\
Curva de sobrevida & 0,175 & Teste de Wilcoxon \\
\hline & & \\
\hline
\end{tabular}


Manifestações clínicas digestivas foram verificadas em $59,6 \%$ dos pacientes, e o balanço de gordura nas fezes estava alterado em $67,9 \%$ destes, indicando uma incidência de insuficiência pancreática abaixo dos dados encontrados na literatura4,7,8. A menor incidência de insuficiência pancreática, comparada à encontrada nos Estados Unidos e na Europa, pode decorrer da menor prevalência da mutação $\Delta \mathrm{F} 508$ no Brasil, a qual está associada à presença desta insuficiência9-14.

No presente estudo e em outro estudo realizado no Brasil $^{5}$, a incidência de íleo meconial foi, respectivamente, de 5,8 e 1,9\% - muito abaixo dos valores citados na literatura, que variam de 15 a $20 \%{ }^{15,16}$. Uma das hipóteses para a menor incidência de íleo meconial no Brasil pode ser a de que os pacientes fibrocísticos morrem no primeiro ano de vida, antes de fazer o diagnóstico.

A incidência de diabetes melito foi semelhante à de outros trabalhos da literatura 4,17,18.

Em relação à idade do início dos sintomas, 72,7\% dos pacientes iniciaram algum tipo de manifestação clínica antes dos 6 meses de idade, com mediana de 3 meses. Isso demonstra que o início é precoce e serve de alerta para a possibilidade de diagnóstico de FC. Três pacientes iniciaram os sintomas após os 10 anos de idade, e um deles após os 20 anos. Esse fato evidencia a importância do diagnóstico em pacientes que começam a ter sintomas respiratórios mais tardiamente ${ }^{19}$

A mediana de idade no diagnóstico foi de 2 anos e 4 meses, e o diagnóstico foi realizado após os 10 anos de idade em $10 \%$ dos casos. Dados do Registro Latino-americano de Fibrosis Quística ${ }^{6}$, onde estão incluídos também os registros do Brasil, mostram que a média de idade ao diagnóstico foi de 4,2 anos. Por outro lado, dados da Cystic Fibrosis Foundation de $2002^{4}$ revelam que a mediana da idade no diagnóstico nos Estados Unidos é de 6 meses, evidenciando que a América Latina como um todo necessita aumentar os esforços para realizar o diagnóstico precoce da FC, como ressalta a Organização Mundial de Saúde ${ }^{1}$.

Nesta casuística, a diferença entre a mediana da idade no diagnóstico e a mediana da idade de início dos sintomas foi de 2 anos e 1 mês. Mitchell et al. ${ }^{20}$ encontraram tempo médio de 3 anos entre o início dos sintomas e o diagnóstico. No Brasil, os estudos de Maróstica ${ }^{21}$ e Espinoza22 mostraram que essa diferença foi de 7 meses em pacientes pediátricos e de 10,7 anos em maiores de 15 anos, respectivamente.

A FC, apesar de não ter cura, apresenta melhora significativa com o tratamento sintomático ${ }^{23}$. Trabalhos recentes têm demonstrado que o diagnóstico precoce diminui a morbidade ${ }^{24}$ e também têm destacado a importância de se iniciar precocemente o tratamento da insuficiência pancreática e da desnutrição, bem como a fisioterapia respiratória para manter as vias aéreas desobstruídas ${ }^{25}$. Um estudo demonstrou que a infecção por $P$. aeruginosa é o principal fator de risco para morbidade e mortalidade, destacando que o diagnóstico precoce serviria para intervenções no início da colonização por esse agente, contribuindo para um melhor prognóstico ${ }^{26}$; outro estudo ressalta, ainda, a importância de se tratar agressivamente a $P$. aeruginosa quando esta for isolada pela primeira vez ${ }^{27}$.

Atualmente, a maioria dos estados do Brasil não realiza triagem neonatal para FC, e sua realização gera controvérsias. O custo da implantação desta triagem em uma região com incidência da doença de $1: 5.000$ seria de $R \$ 25.000,00$ para cada caso novo. Os pontos negativos para a realização da triagem incluem o custo elevado, os resultados falsopositivos e falso-negativos e o fato de revisões recentes não terem demonstrado diferença na evolução da FC entre pacientes diagnosticados por triagem neonatal e aqueles que tiveram diagnóstico após o início dos sintomas 28,29 . Comenta-se que uma maior divulgação da FC para os pediatras, aliada ao acesso facilitado ao diagnóstico por eletrólitos no suor, seria uma alternativa mais plausível. 0 argumento do custo pode ser questionado, pois um recente trabalho demonstrou que o custo por diagnóstico realizando a triagem neonatal é inferior ao custo por diagnóstico quando esta não é utilizada ${ }^{30}$. Outro trabalho, na França, demonstrou que pacientes com o diagnóstico feito por triagem neonatal tiveram evolução melhor que aqueles com o diagnóstico realizado após o início dos sintomas ${ }^{31}$. Sabese que a demora na identificação e tratamento da Pseudomonas dificulta sua erradicação ${ }^{27}$. Exames sorológicos podem detectar a colonização por Pseudomonas 6 a 12 meses antes da positivação da cultura ${ }^{32}$. Desta forma, o diagnóstico por triagem neonatal levaria à realização de sorologias de rotina, possibilitando a detecção mais precoce e a tentativa de erradicação mais eficiente desta bactéria. Existe também a esperança de disponibilizar para os pacientes fibrocísticos uma vacina contra Pseudomonas aeruginosa. Com isso, a triagem neonatal será menos controversa, pois sem ela se perderia a possibilidade, para alguns pacientes, de realizar a vacina antes da colonização. Por último, lembramos que a triagem neonatal possibilitará o aconselhamento genético dos pais antes que ocorra uma nova gravidez.

Quando a triagem neonatal não é realizada, o diagnóstico precoce pode indicar maior gravidade. Um estudo brasileiro verificou que a combinação de baixo ES, baixo peso ao nascimento e baixa idade ao diagnóstico são indicativos de pior prognóstico ${ }^{33}$.

Nos 10 anos de acompanhamento, 17,3\% dos pacientes foram a óbito. A mediana da idade do óbito foi de 7,8 anos, e a sobrevida mediana após o diagnóstico, na época do encerramento do estudo, foi de 18,4 anos. Nos Estados Unidos, a sobrevida mediana aumentou de 1 ano em 1940 para 20 anos em 198034. Em 1990, a sobrevida dos fibrocísticos no Canadá e nos Estados Unidos era de 30,9 e 27,6 anos, respectivamente 35 . Outro estudo cita que a expectativa de vida para pacientes com FC aumentou de 8 anos em 1970 para 29,5 anos em $1998^{36}$. Segundo dados da Cystic Fibrosis Foundation, a expectativa de vida atual nos Estados Unidos é de $31,6^{4}$ anos. A mortalidade nos Estados Unidos entre 1985 e 1999 para pacientes com idade entre 2 e 15 anos diminuiu entre 45 e $70 \%{ }^{37}$. Outro estudo apresenta dados ainda mais otimistas e considera que, atualmente, para um recém-nascido com FC, a expectativa de vida é de mais de $40 \operatorname{anos}^{38}$. 
Em outro estudo no Brasil, a sobrevida mediana, após o nascimento, de uma coorte de 111 pacientes diagnosticados entre 1970 e 1994 foi de 12,6 anos $^{39}$.

A baixa sobrevida em nosso país quando comparada à de países desenvolvidos é muito preocupante e demonstra níveis que ocorriam 20 anos atrás nesses países. Acreditamos que alguns fatores possam estar contribuindo para esse fato. Inicialmente, a demora no diagnóstico pode fazer com que os pacientes iniciem o tratamento em uma fase onde já houve deterioração pulmonar, e talvez se tenha perdido a oportunidade de tratar precocemente as exacerbações por $P$. aeruginosa. Além disso, na época do diagnóstico, 70 e $56,6 \%$ dos nossos pacientes apresentavam peso e estatura abaixo do percentil 10, respectivamente, valores maiores do que os relatados encontrados na literatura (em torno de 42 a $44 \%{ }^{4,40}$ ). A desnutrição causada pela demora em iniciar o tratamento deve também contribuir para uma deterioração mais rápida, pois tem-se demonstrado a relação entre desnutrição e piora da função pulmonar ${ }^{41}$. Outro fator que pode estar contribuindo é a pouca quantidade de centros especializados no tratamento de FC. Como cada centro abrange uma área e uma população muito grande, a dificuldade dos pacientes em procurarem esses centros pode estar contribuindo para que o tratamento e acompanhamento fiquem prejudicados. Segundo as diretrizes da Cystic Fibrosis Foundation, cada paciente deveria realizar quatro visitas por ano, o que não é possível para muitos de nossos pacientes. O aumento do número de centros de tratamento de $\mathrm{FC}$ e/ou a melhoria do sistema de transporte desses pacientes poderiam contribuir para um acompanhamento mais freqüente e para a redução da morbidade e mortalidade.

Trabalhos recentes demonstram pior evolução nos pacientes fibrocísticos com nível socioeconômico inferior. Schechter et al. verificaram risco de morrer 3,65 vezes maior nos fibrocísticos mais pobres, os quais também apresentavam valores de função pulmonar e estado nutricional mais deteriorados 42 . Esses achados foram confirmados por uma amostra muito grande de pacientes da Cystic Fibrosis Foundation 4 . Um estudo recente demonstrou que pacientes com melhor nível socioeconômico apresentam risco $40 \%$ menor em relação à mortalidade quando comparados com pacientes de nível socioeconômico 43 inferior. Desta forma, a maior morbidade e mortalidade de nossos pacientes pode ter relação com um baixo nível socioeconômico.

A dosagem de cloro no suor foi menor que $60 \mathrm{mEq} / \mathrm{l}$ em 11 pacientes $(10,6 \%)$. Esta porcentagem é maior que os valores citados na literatura - alguns autores referem que apenas $2 \%$ dos pacientes fibrocísticos apresentam cloro no suor menor que $60 \mathrm{mEq} / \mathrm{I}^{44,45}$.

Desses 11 pacientes, seis (três masculinos) tiveram três ou mais dosagens de cloro no suor menores que $60 \mathrm{mEq} / \mathrm{I}$ e diagnóstico confirmado pela identificação de duas mutações. Todos eram caucasóides, apresentavam balanço de gordura fecal normal e sintomas respiratórios, mas não apresentavam sintomas digestivos. Todos estavam colonizados cronicamente por Staphylococcus aureus e Pseudo- monas aeruginosa, cinco por Pseudomonas aeruginosa mucosa e dois por Burkholderia cepacia. O estudo genético evidenciou quatro pacientes $\Delta F 508$ homozigotos e dois $\Delta \mathrm{F} 508 / \mathrm{N} 1303 \mathrm{~K}$

Os outros cinco pacientes que tiveram dosagem de cloro no suor menor que $60 \mathrm{mEq} / \mathrm{l}$ em nossa casuística apresentavam sintomas respiratórios e digestivos compatíveis com FC, melhoraram com a utilização de enzimas pancreáticas, eram colonizados por Pseudomonas aeruginosa e tinham uma mutação $\Delta \mathrm{F} 508$; desta forma, foram considerados fibrocísticos e receberam o tratamento convencional.

O diagnóstico de FC com valores de cloro e sódio normais no suor tem sido verificado também por outros autores 46,47 . Nesses casos, deve-se tentar confirmar o diagnóstico pela identificação de duas mutações para FC ou pela medida da diferença do potencial nasal ${ }^{48-50}$ (este exame ainda não é realizado em nosso serviço). Quando não se consegue estabelecer o diagnóstico pelos métodos sugeridos, este deve basear-se no julgamento clínico ${ }^{48}$, como foi o caso desses cinco pacientes citados.

Um total de $76 \%$ dos pacientes estavam colonizados por Pseudomonas aeruginosa, índice próximo ao encontrado na literatura 4,51 .

Em relação a Pseudomonas aeruginosa mucosa e Staphylococcus aureus, teve-se uma incidência de colonização de 53 e $80 \%$, respectivamente, semelhante aos valores encontrados em outros estudos $4,7,22,52$.

A presença de Burkholderia cepacia em cinco pacientes $(5,2 \%)$ é um fato preocupante, pois a colonização por esta bactéria leva a um prognóstico ruim e diminui a sobrevida 53 , sendo que um estudo demonstrou que essa diminuição chega a uma década quando comparada à de pacientes infectados apenas por Pseudomonas aeruginosa ${ }^{54}$. Valores de prevalência de Burkholderia cepacia em geral variam de 5 a $15 \% 4,21,55$, sendo maior em algumas regiões, como em Ontário, no Canadá, onde chega a $22 \% 56$.

Dados anteriores do Brasil $^{21}$ relatam que $10,7 \%$ dos pacientes apresentam distúrbio ventilatório restritivo, 25\% distúrbio ventilatório obstrutivo e 17,9\% distúrbio ventilatório misto, valores também muito semelhantes aos encontrados em nossa casuística.

Realizamos a genotipagem em $92,31 \%$ de nossos pacientes, o que representa uma boa porcentagem quando comparada à Cystic Fibrosis Foundation, onde $81,4 \%$ dos pacientes foram genotipados ${ }^{4}$. Em nossa casuística, a mutação $\Delta \mathrm{F} 508$ estava presente em $50 \%$ dos 192 cromossomos estudados. As outras mutações foram encontradas nas seguintes porcentagens: G542X $(4,17 \%)$, N1303K $(2,08 \%)$, G551D $(1,04 \%), \operatorname{R} 553 \mathrm{X}(0,52 \%), \mathrm{W} 1282 \mathrm{X}$ $(0,52 \%)$.

Também no Brasil, Raskin et al. ${ }^{57}$ relataram que as mutações mais freqüentes na população brasileira são: $\Delta \mathrm{F} 508(47 \%), \mathrm{G} 542 \mathrm{X}(5,5 \%), \mathrm{N} 1303 \mathrm{~K}(2,6 \%), \mathrm{R} 553 \mathrm{X}$ $(0,8 \%)$ e G551D $(0,2 \%)$. Esses autores citam ainda que $26 \%$ dos pacientes com FC são homozigotos para a mutação $\Delta \mathrm{F} 508$. 
Nos Estados Unidos 58 , as mutações mais freqüentes encontradas foram: $\Delta \mathrm{F} 508(66 \%), \mathrm{G} 542 \mathrm{X}(2,4 \%), \mathrm{G} 551 \mathrm{D}$ $(1,6 \%), \operatorname{N1303K}(1,3 \%), \mathrm{W} 1282 \mathrm{X}(1,2 \%)$ e R533X $(0,7 \%)$. Na França 59 , a mutação $\Delta \mathrm{F} 508$ estava presente em $67,9 \%$ dos cromossomos de uma população de 2.666 pacientes com FC; as outras mutações mais freqüentes foram $\mathrm{G} 542 \mathrm{X}$ $(2,5 \%), N 1303 \mathrm{~K}(2 \%), 1717-1 \mathrm{G} \rightarrow \mathrm{A}(1,2 \%), \operatorname{R} 553 \mathrm{X}(0,8 \%)$ e G551D $(0,7 \%)$. Na Argentina, a mutação $\Delta \mathrm{F} 508$ está presente em $66 \%$ dos cromossomos de pacientes fibrocísticos ${ }^{60}$, e no México, 7,2\% dos cromossomos de pacientes com FC apresentam a mutação G542X61.

A menor incidência da mutação $\Delta \mathrm{F} 508$ em nosso meio quando comparada aos dados dos Estados Unidos, França e Argentina provavelmente ocorre devido à grande miscigenação de raças no Brasil.

Cerca de $60 \%$ dos pacientes apresentavam ES excelente ou bom, e apenas $15,7 \%$ apresentavam escore moderado ou grave. Henry et al. 62 , analisando 60 pacientes, referiram que a média do ES foi de 78,87 .

Nesta análise, o ES apresentou correlação estatisticamente significativa com colonização por Pseudomonas aeruginosa, colonização por Pseudomonas aeruginosa mucosa, capacidade vital forçada, volume expiratório forçado no primeiro segundo, saturação transcutânea da hemoglobina pelo oxigênio, número de exacerbações infecciosas no último ano de acompanhamento, indicação de utilização de dornase alfa, indicação de programa regular de fisioterapia respiratória e indicação de oxigenoterapia domiciliar. Como esses parâmetros se relacionam individualmente com a gravidade do quadro clínico, considerou-se que o ES é um bom método de avaliação geral da gravidade. Outro trabaIho brasileiro evidenciou que o ES apresenta uma correlação positiva com o volume expiratório forçado no primeiro segundo63.

Nesta casuística, a presença da mutação $\Delta \mathrm{F} 508$ estava associada com alteração no balanço de gordura e insuficiência pancreática. Esses dados estão de acordo com a literatura internacional, onde vários autores demonstraram que a mutação $\Delta$ F508 está associada à presença de insuficiência pancreática9-13.

Nesta casuística, os pacientes $\Delta \mathrm{F} 508$ homozigotos não apresentaram nenhuma característica diferente quando comparados aos heterozigotos para esta mutação. Na literatura há poucos trabalhos comparando esses dois grupos. Farrell \& Koscik ${ }^{64}$ relataram que os níveis de cloro no suor são iguais nos dois grupos.

Em conclusão, as características clínicas e laboratoriais dos 104 pacientes fibrocísticos estudados foram semelhantes às descritas na população fibrocística de outros países, com exceção de: insuficiência pancreática (menos freqüente), antecedente de íleo meconial (menos freqüente), mediana da idade no diagnóstico (maior), mediana da idade do óbito e sobrevida após o diagnóstico (menores quando comparados aos dados dos Estados Unidos e Canadá), desnutrição na época do diagnóstico (maior que a relatada em outros países), porcentagem de pacientes com dosagem de cloro no suor normal (maior do que a relatada na literatura internacional), incidência da mutação $\Delta$ F508 (me- nor quando comparada aos dados dos Estados Unidos e da Europa).

Nossos resultados também permitem concluir que, em pacientes que apresentam clínica sugestiva de FC, a dosagem de cloro no suor $<60$ mEq/I não exclui esse diagnóstico, devendo ser realizado o estudo genético para tentar definir o diagnóstico.

Nossos dados permitem inferir que esforços para um diagnóstico precoce e maior oportunidade de tratamento necessitam ser dirigidos aos pacientes com FC.

\section{Agradecimentos}

Os autores agradecem ao Prof. Dr. Fernando Antonio de Abreu e Silva, da UFRGS, pelas oportunas sugestões para a realização deste trabalho.

\section{Referências}

1. World Health Organization and International Cystic Fibrosis (Mucoviscidosis) Association - Implementation of cystic fibrosis services in developing countries: memorandum from a joint WHO/ICF (M)A meeting. Bulletin of the World Health Organization. 1997;75(1):10.

2. Gibson LE, Cooke RE. A test for concentration of electrolytes in sweat in cystic fibrosis of the pancreas utilizing pilocarpine by iontophoresis. Pediatrics. 1959;23:545-9.

3. Rommens JM, Kerem B, Greer W, Chang P, Tsui LC, Ray P. Rapid non radioactive detection of the major cystic fibrosis mutation. Am J Hum Genet. 1990;46:395-6.

4. Cystic Fibrosis Foundation, Patient Registry. Bethesda, Maryland: 2002 Annual Data Report; 2003.

5. Camargos PAM, Guimarães MDC, Reis FJC. Prognostic aspects of cystic fibrosis in Brazil. Ann Trop Pediatr. 2000;20:287-91.

6. Registro Latino-Americano de Fibrosis Quística (REGLAFQ). Informe del cuarto año. Buenos Aires; 1993. p. 21.

7. Huang N, Schidlow D, Szatrowski T, Palmer J, Laraya-Cuasay L, Yeung $W$, et al. Clinical features, survival rate and prognostic factors in young adults with cystic fibrosis. Am J Med. $1987 ; 2: 871-9$

8. Welsh MJ, Tsui LC, Boat TF, Beaudet AL. Cystic fibrosis. In: Scriver CR, Beaudet AL, Sly WS, Valle D. The Metabolic and Molecular Bases of Inherited Disease. 7th ed. New York: McGraw-Hill; 1995. p. 3799-3876.

9. Kerem E, Corey M, Kerem B, Rommens JM, Markiewicz D, Levison $\mathrm{H}$, et al. The relationship of the most common mutation $\Delta$ F508. N Engl J Med. 1990;323:1517-22.

10. Borgo G, Mastella G, Gasparini P, Zoranello A, Doro R, Pignatti PF. Pancreatic function and genetic $\Delta$ F508 in cystic fibrosis. J Med Genet. 1990;27:665-9.

11. Santis G, Osborne L, Knight RA, Hodson ME. Independent genetic determinants of pancreatic and pulmonary status in cystic fibrosis. Lancet. 1990;336:1081-4.

12. Campbell PW III, Phillips JA III, Krishnamani MR, Maness KJ, Hajinski TA. Cystic fibrosis: relationship between clinical status and $\Delta$ F508 deletion. J Pediatr. 1991;118:239-41.

13. Johansen HK, Nir M, Hoib YN, Koch C, Schwartz M. Severity of cystic fibrosis in patients homozygous and heterozygous for $\Delta$ F508 mutation. Lancet. 1991;337:631-4.

14. McKone EF, Emerson SS, Edwards KL, Aitken ML. Effect of genotype on phenotype and mortality in cystic fibrosis: a retrospective cohort study. Lancet. 2003;361:1671-6.

15. Cystic Fibrosis Foundation. Clinical Practice Guidelines for Cystic Fibrosis; 1997.

16. Park RW, Grand RJ. Gastrointestinal manifestations of cystic fibrosis: a review. Gastroenterology. 1981;81:1143.

17. Finkelstein SM, Wielinski CL, Elliott GR. Diabetes mellitus associated with cystic fibrosis. J Pediatr. 1988;112:373.

18. Rosenecker J, Eichler I, Kuhn L, Harms HK, Von de Hardt J. Genetic determination of diabetes mellitus in Danish CF patients: prevalence and late diabetic complications. Acta Paediatr. $1994 ; 83: 72-7$. 
19. Bargon J, Rickmann J, Jacobi V, Straub R, Arnemann J, Wagner TO. Cystic fibrosis: initial diagnosis in a 39 year-old patient. Med Klin. 2000;95:697-700.

20. Mitcell-Heggs $P$, Mearns $M$, Batten JC. Cystic fibrosis in adolescents and adults. Quarter J Med New Series. 1976;XLV: 479-504.

21. Maróstica PJC. Avaliação pneumológica de pacientes portadores de fibrose cística: sua relação com grupos genéticos [tese]. Porto Alegre, Universidade Federal do Rio Grande do Sul; 1995.

22. Domec Espinoza MPS. Fibrose cística em jovens e adultos do Hospital das Clínicas da Unicamp [dissertação]. Campinas, Universidade Estadual de Campinas; 1998.

23. Tauber E, Eichler I, Gartner C, Halmerbauer G, Gotz M, Rath R, et al. Improvements of lung function in cystic fibrosis. Pediatr Pulmonol. 2002;33:263-8.

24. Wang SS, O'Leary LA, Fitzsimmons SC, Khoury MJ. The impact of early cystic fibrosis on pulmonary function in children. J Pediatr. 2002;141:804-10.

25. Wagener JS, Headley AA. Cystic fibrosis: current trends in respiratory care. Respir Care. 2003;48:234-45.

26. Emerson J, Rosenfeld M, McNamara S, Ramsey B, Gibson RL. Pseudomonas aeruginosa and other predictors of mortality and morbidity in young children with cystic fibrosis. Pediatr Pulmonol. 2002;34:91-100.

27. Bush A. Decisions facing the cystic fibrosis clinician at the first isolation of Pseudomonas aeruginosa. Paediatr Respir Rev. 2002;3:82-8.

28. Merelle ME, Nagelkerke AF, Lees CM, Dezateaux C. Newborn screening for cystic fibrosis. Cochrane Database Syst Rev. 2001;3:CD001402.

29. Farrell PM, Li Z, Kosorok MR, Laxova A, Green CG, Collins J, et al. Bronchopulmonary disease in children with cystic fibrosis after early or delayed diagnosis. Am J Respir Crit Care Med. 2003;168:1100-8.

30. Lee DS, Rosenberg MA, Peterson A, Makholm L, Hoffman G, Laessig $\mathrm{RH}$, et al. Analysis of the costs of diagnosing cystic fibrosis with a newborn screening program. J Pediatr. 2003;142:617-23.

31. Siret D, Bretaudeau G, Branger B, Dabadie A, Dagorne M, David $V$, et al. Comparing the clinical evolution of cystic fibrosis screened neonatally to that of cystic fibrosis diagnosed from clinical symptoms: a 10-year retrospective study in a French region (Brittany). Pediatr Pulmonol. 2003;35:342-9.

32. West SE, Zeng L, Lee BL, Kosorok MR, Rock MJ, Splaingard MJ, et al. Respiratory infections with Pseudomonas aeruginosa in children with cystic fibrosis: early detection by serology and assessment of risk factors. JAMA. 2002;287:2958-67.

33. Oliveira MC, Reis FJ, Oliveira EA, Colosimo EA, Monteiro AP, Penna FJ. Prognostic factors in cystic fibrosis in a single center in Brazil: a survival analysis. Pediatr Pulmonol. 2002;34:3-10.

34. Maclusky I, Levison H. Cystic fibrosis. In: Chernick V, Boat TE. Kendig's Disorders of the Respiratory Tract in Children. Philadelphia: Saunders; 1990. p. 692-729.

35. Kerem E, Reisman J, Corey M, Canny GJ, Levison H. Prediction of mortality in patients with cystic fibrosis. $N$ Engl J Med. 1992;326:1187-91.

36. Bolyard DR. Sexuality and cystic fibrosis. Am J Matern Child Nurs. 2001;26:39-41.

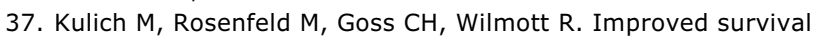
among young patients with cystic fibrosis. J Pediatr. 2003; 142:631-6.

38. Doull IJ. Recent advances in cystic fibrosis. Arch Dis Child. 2001;85:62-6.

39. Reis FJC, Camargos PAM, Rocha SF. Survival analysis for cystic fibrosis in Minas Gerais State, Brazil. J Trop Pediatr. 1998;44: 329-31.

40. Farrell PM, Kosorok MR, Laxova A, Shen G. Nutritional benefits of neonatal screening for cystic fibrosis. $N$ Engl J Med. 1997;337:963-9.

41. Zemel BS, Jawad AF, FitzSimmons S, Stallings VA. Longitudinal relationship among growth, nutritional status and pulmonary function in children with cystic fibrosis: analysis of the Cystic Fibrosis Foundation National Patient Registry. J Pediatr. 2000;137:374-80.

42. Schechter MS, Shelton BJ, Margolis PA, Fitzsimmons SC. The association of socioeconomic status with outcomes in cystic fibrosis in the United States. Am J Respir Crit Care Med. 2001;163:1331-7.
43. O'Connor GT, Quinton HB, Kahn R, Robichaud P, Maddock J, Lever $\mathrm{T}$, et al. Case-mix adjustment for evaluation of mortality in cystic fibrosis. Pediatr Pulmonol. 2002;33:99-105.

44. Huff DS, Huang NN, Arey JB. Atypical cystic fibrosis of the pancreas with normal levels of sweat chloride and minimal pancreatic lesions. J Pediatr. 1979;94:237.

45. Stewart B, Zabner J, Shuber AP, Welsh MJ, Mccray Jr. PB. Normal sweat chloride values do not exclude the diagnosis of cystic fibrosis. Am J Respir Crit Care Med. 1995;151:899-903.

46. Desmarquest P, Feldman N, Tamalat A, Boule M, Fauroux B, Tournier G, et al. Genotype analysis and phenotypic manifestations of children with intermediate sweat chloride test results. Chest. 2000;118:1591-7.

47. Lebecque $P$, Leal $T$, De Boeck C, Jaspers $M$, Cuppens $H$, Cassiman J. Mutations of the cystic fibrosis gene and intermediate sweat chloride levels in children. Am J Respir Crit Care Med. 2002; 165:757-61.

48. Rosentein BJ, Cuting GR. The diagnosis of cystic fibrosis: a consensus statement. Cystic Fibrosis Foundation Consensus Panel. J Pediatr. 1998;132:589-95.

49. Rosentein BJ. What is a cystic fibrosis diagnosis? Clin Chest Med. 1998; 19:423-41.

50. Ratjen F, Döring G. Cystic fibrosis. Lancet. 2003;361:681-9.

51. Burns JL, Gibson RL, Mcnamara S, Yim D, Emerson J, Rosenfeld $M$, et al. Longitudinal assessment of Pseudomonas aeruginosa in young children with cystic fibrosis. J Infect Dis. 2001;183: 444-52.

52. Dornelas EC, Fernandes MIM, Galvão LC, Silva GA. Estudo do quadro pulmonar de pacientes com fibrose cística. J Pediatr (Rio J). 2000;76:295-99.

53. Soni R, Marks G, Henry DA, Robison M, Moriaty C, Parsons S, et al. Effects of Burkholderia cepacia infection in the clinical course of patients with cystic fibrosis: a pilot study in a Sydney clinic. Respirology. 2002;7:241-5.

54. Jones AM, Dodd ME, Webb AK. Burkholderia cepacia: current clinical issues, environmental controversies and ethical dilemmas. Eur Respir J. 2001;17:295-301.

55. Lewin LO, Byard PJ, Davis PB. Effect of Pseudomonas cepacia colonization on survival and pulmonary function of cystic fibrosis patients. J Clin Epidemiol. 1990;43:125-31.

56. Speert DP, Henry D, Vandamme P, Corey M, Mahenthiralingam E. Epidemiology of Burkholderia cepacea complex in patients with cystic fibrosis, Canada. Emerg Infec Dis. 2002;8:181-7.

57. Raskin S, Philips III JA, Krishnamani MRS, Jones C, Parker RA, Rozov T. DNA analysis of cystic fibrosis in Brazil by direct PCR amplification from guthrie cards. Am J Med Gen. 1993;46:665-9.

58. Cystic Fibrosis Genetic Analysis Consortium - Population variation of common cystic fibrosis mutations. Human Mutation. 1994; $4: 167-77$.

59. Guilloud-Batalie M, De Crozes D, Rault G, Degioanni A, Feingold J. Cystic fibrosis mutations: report from the French Registry. The Clinical Centers of the CF. Hum Hered. 2000;50:142-5.

60. Saleh MC, Botelli A, Melano De Botelli M, Rezzonico CA, Argaraña CE. Cystic fibrosis: frequency of delta F508 and G542X mutations in Cordoba, Argentina. Medicina (B. Aires). 1996;56:14-6.

61. Villarreal MT, Chavez M, Lezana JL, Cuevas F, Carnevale A, Codova $E$, et al. G542X mutation in Mexican cystic fibrosis patients. Clin Genet. 1996;49:54-6.

62. Henry RL, Mellis CM, Petrovic L. Mucoid Pseudomonas aeruginosa is a marker of poor survival in cystic fibrosis. Pediatr Pulmonol. $1992 ; 12: 158-61$

63. Assis I, Camargos PAM, Reis FJC, Sulmonett N, Carneiro APS. Assessing correlations between spirometry and ShwachmanKulczycki score in children and adolescents. Pediatr Pulmonol. 2003;36:305-9.

64. Farrell PM, Koscik RE. Sweat chloride concentrations in infants homozygous or heterozygous for $\Delta \mathrm{F} 508$ cystic fibrosis. Pediatrics. $1996 ; 97: 524-8$.

\section{Correspondência:}

Alfonso Eduardo Alvarez

Rua Cel. de Moraes, 317/31

CEP 13075-020 - Campinas, SP

Fones: (19) 3242.8824/3243.7227

Fax: (19) 3243.7227

E-mail: alfonsoea@terra.com.br 